\title{
Formulation, characterisation and flexographic printing of novel Boger fluids to assess the effects of ink elasticity on print uniformity
}

\author{
Miles L. Morgan ${ }^{1} \cdot$ Alexander Holder ${ }^{1}$. Dan J. Curtis ${ }^{1} \cdot$ Davide Deganello $^{1}$ (i) \\ Received: 1 June 2017 / Revised: 9 November 2017 / Accepted: 14 November 2017 / Published online: 7 December 2017 \\ (C) The Author(s) 2017. This article is an open access publication
}

\begin{abstract}
Model elastic inks were formulated, rheologically characterised in shear and extension, and printed via flexography to assess the impact of ink elasticity on print uniformity. Flexography is a roll-to-roll printing process with great potential in the mass production of printed electronics for which understanding layer uniformity and the influence of rheology is of critical importance. A new set of flexo-printable Boger fluids was formulated by blending polyvinyl alcohol and high molecular weight polyacrylamide to provide inks of varying elasticity. During print trials, the phenomenon of viscous fingering was observed in all prints, with those of the Newtonian ink exhibiting a continuous striping in the printing direction. Increasing elasticity significantly influenced this continuity, disrupting it and leading to a quantifiable decrease in the overall relative size of the printed finger features. As such, ink elasticity was seen to have a profound effect on flexographic printing uniformity, showing the rheological tuning of inks may be a route to obtaining specific printed features.
\end{abstract}

Keywords Boger fluid · Extensional flow · Flexographic printing · Elastic properties

\section{Introduction}

Functional printing is an emergent mode of controlled deposition that offers exciting prospects in electronic device fabrication. In particular, roll-to-roll printing techniques such as flexography have tremendous potential for the production of low cost, high volume printed electronics. As an established technique in the packaging industry (Kipphan 2001), flexography enables printing at high speeds with multi-layer device manufacture facilitated by the continuous nature of the roller-based process. Additionally, the flexographic method can print thin patterned films on flexible substrates at ambient temperatures with little waste and has been utilised in the fabrication of transistors (Yan et al. 2009), conductive grids (Deganello et al. 2010b), biosensors (Benson et al. 2015), gas sensors (Lloyd et al. 2013) and photovoltaics (Rand et al. 2007; Baker et al. 2014). However, due to the tight tolerances required for device functionality and the rheological complexity of functional printing inks, a better understanding of the

$\triangle$ Davide Deganello

D.Deganello@swansea.ac.uk

1 College of Engineering, Swansea University, Bay Campus, Fabian Way, Crymlyn Burrows, Swansea, SA1 8EN, UK influence of ink rheology on print quality is essential for the large-scale transition from graphics to functional rollto-roll printing. Relations between ink viscoelasticity and printability have been reported for techniques including inkjet (Tuladhar and Mackley 2008; Morrison and Harlen 2010), offset lithography (Pangalos et al. 1985), gravure (Sunilkumar and Rothstein 2017) and screen printing (Somalu et al. 2013); however, works studying and exploiting the effects of ink viscoelasticity on the quality of flexographic prints are lacking, especially considering its potential applications.

Flexography, illustrated in Fig. 1, is a relief process that employs a photopolymer printing plate on which the desired printing pattern is raised. Ink is supplied to the printing plate via a metered anilox roll-a cylinder finely engraved with many small cells. The size and frequency of these cells control the volume of ink transferred to the patterned printing plate before it is subsequently transferred to the substrate. The formulation of functional flexographic ink containing conductive particles, in contrast to traditional pigmented ink for graphics applications, therefore enables the printing of bespoke conductive configurations. However, challenges remain in the pursuit of mass-produced printed electronics which have a greater requirement for high print resolution, registration and uniformity (Deganello et al. 2010a; Lee 


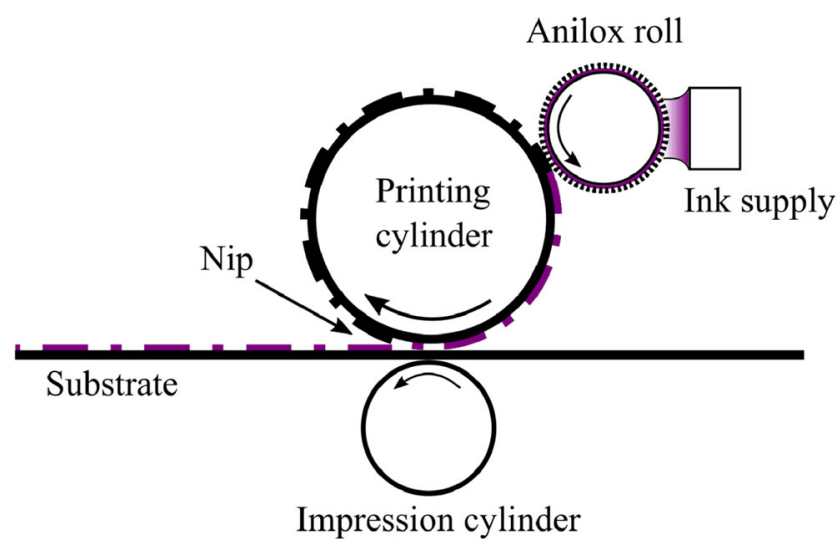

Fig. 1 Illustration of the flexographic process

et al. 2013; Schmidt et al. 2010). Poor uniformity can be particularly problematic, affecting device performance and even causing terminal issues such as short circuits. A salient manifestation of non-uniformity in flexography is the phenomenon of viscous fingering (Sauer et al. 2011; Wang et al. 2014) which appears in the form of fingerlike features along the print and has been attributed to the Taylor-Saffman instability that occurs when a fluid is displaced by a less viscous fluid (Saffman and Taylor 1958). Ideally observed in a Hele-Shaw cell, competition between surface tension and viscous forces leads to finger formation of a characteristic wavelength dependent on material properties and process parameters (Lindner and Wagner 2009). In flexography, this instability occurs as the inked substrate and printing plates are separated, creating a dynamic meniscus axially along the printing cylinder between air and the ink, which is being subjected to both shear and extensional stresses (Sauer et al. 2011). Furthermore, functional printing inks typically contain a considerable amount of polymer resin that acts to bind conductive particles of various materials and conformations in conducting networks. These constituents often lead to the exhibition of viscoelastic and non-Newtonian characteristics which have been seen to affect the size of fingers in a Hele-Shaw cell (Lindner et al. 2002; Lindner and Wagner 2009). Elasticity has been specifically studied in this context using Boger fluids, with it encouraging tipsplitting at high velocities (Vlad and Maher 2000) and causing the formation of narrower fingers (Huzyak and Koelling 1997; Malhotra and Sharma 2014), in contrast to observations by Lindner et al. (2002) of wider fingers with flexible polymers.

While work has been carried out to understand instabilities in roller processes such as forward roll coating (Fernando and Glass 1988; Zevallos et al. 2005; Han et al. 2009; Gaskell et al. 1998), very little has focussed on their incidence in flexographic printing. The focus of the literature is therefore primarily on the dynamics of model fluids between two rotating rolls in the absence of substrate. For Newtonian fluids, the wavelength of viscous fingers in flexography is thought to decrease with capillary number, $C a=\eta v / \sigma$ where $v$ is process velocity and $\eta$ and $\sigma$ are fluid shear viscosity and surface tension, respectively (Sauer et al. 2011; Voß 2002); however, analysis with respect to $C a$ does not take into account the viscoelastic and non-Newtonian characteristics of the material. Destabilisation of flow caused by liquid elasticity in forward roll coating is thought to result in the onset of ribbing - a similar phenomenon to the here explored viscous fingering - at a lower capillary number (Bauman et al. 1982; Fernando and Glass 1988; Zevallos et al. 2005). The occurrence of such phenomena as cavitation, misting and in particular filamentation (Fernando et al. 2000), facilitated by extensional flows, should also be considered. Studies regarding elasticity in roller processes suggest it leads to smaller filaments (Ercan 2001) and that they are longer and more stable (Fernando and Glass 1988). These effects would likely impact the formation and printing of viscous fingers in flexography and subsequently the uniformity of resulting prints; however, this has not been reported upon. Viscous, elastic and surface tension forces play a fundamental role in many other phenomena that have been extensively researched including drop impact (Crooks et al. 2001), jetting (Hoath et al. 2014) and bubble collapse (Sabadini et al. 2014) with interfacial viscoelastic effects also being exploited by Spaid MA and Homsy GM (1996) to increase the stability of dynamic contact lines. Such rheological tuning of inks and process parameters could be of much benefit to the advancement of large-scale functional printing.

Herein, by specially formulating new printable Boger fluid inks of similar shear viscosity, the effect of ink elasticity on flexographic print uniformity has been studied in a real printing scenario, with ink characterisation in both shear and extension.

\section{Materials and methodology}

\section{Ink formulation}

A model printing ink should not only exhibit strict rheological traits but also have adequate drying performance and a suitable surface tension to prevent excessive wetting or dewetting on the substrate, while in the current work also enabling the observation of viscous fingering. Model elastic fluids such as polyethylene glycol and polyethylene oxide solutions (Dontula et al. 1998) have relatively high surface tensions at low viscosities (Yu et al. 2006) while oil-based polystyrene solutions (Anna and Mckinley 2001) exhibit difficulties upon drying and thus are not suitable 
Table 1 Solid weight contents of formulated Boger fluid inks

\begin{tabular}{llll}
\hline Fluid & PVA $(\mathrm{wt} \%)$ & PAM $(\mathrm{wt} \%)$ & Dye $(\mathrm{wt} \%)$ \\
\hline $\mathrm{N}$ & 10 & 0 & 0.05 \\
E1 & 9.75 & 0.0125 & 0.05 \\
E2 & 9.55 & 0.025 & 0.05 \\
E3 & 8.7 & 0.1 & 0.05 \\
\hline
\end{tabular}

for printing. As such, this work presents a new class of water-based printable Boger fluids that may be formulated to a range of elasticities and viscosities. These fluids were formulated by blending polyvinyl alcohol (PVA) of molecular weight $20-30 \times 10^{3} \mathrm{~g} / \mathrm{mol}$ with polyacrylamide (PAM) of molecular weight $5-6 \times 10^{6} \mathrm{~g} / \mathrm{mol}$ from Acros Organics. PVA and PAM have been shown to be miscible over a range of concentrations (El-din et al. 2003) however constitute no reported flexographic ink or Boger fluid known to the authors. Solutions of each polymer in deionised water were mixed at various concentrations to produce blends of a similar shear viscosity. Table 1 shows the final composition of each ink and Table 2 displays their measured physical properties. In order to colour the solutions, E122 azorubine dye (FastColours LLP) was dissolved in water and added to each at a solid content of $0.05 \mathrm{wt} \%$.

\section{Ink characterisation}

Shear viscosity measurements were carried out on a Malvern Bohlin rotational rheometer with a $40 \mathrm{~mm}, 4^{\circ}$ cone and plate geometry between shear rates of 100 and $0.1 \mathrm{~s}^{-1}$.

Viscoelastic measurements were carried out on a TA Instruments AR-G2 with a $60 \mathrm{~mm}$ aluminium parallel plate geometry. In light of the extensional flows present in flexography, a purpose built capillary breakup extensional rheometer (CaBER) was used to characterise the uniaxial extensional properties of the inks with a plate diameter, $D_{0}$, of $3 \mathrm{~mm}$. An initial aspect ratio of 0.43 was used for all inks with final aspect ratios of 1.1 for ink $\mathrm{N}$ and 1.2 for inks E1, E2 and E3 to ensure surface tension-driven filament formation and breakup. Filament breakup was captured with a FastCam high-speed camera at between 1000 and 4000 frames/s depending on breakup time. From the recorded images, mid-filament diameter $D_{\text {mid }}(t)$ was extracted and used to calculate apparent extensional viscosity $\eta_{E}$ :

$\eta_{E}=(2 X-1) \frac{\sigma}{-d D_{\text {mid }} / d t}$

where $\sigma$ is the ink surface tension and $X$ is a dimensionless variable dependent on the tensile force and radius of the filament (Tuladhar and Mackley 2008). For Newtonian fluid breakup, $X$ is taken to be 0.7127 as determined by Papageorgiou (1995) while for elastic breakup, $X=1$ due to the axial symmetry of the filament (McKinley and Tripathi 2000). Furthermore, elastic filament breakup may be modelled with

$\frac{D_{m i d}(t)}{D_{1}}=\exp \left(-t / 3 \lambda_{E}\right)$

where $D_{1}$ is mid-filament diameter just after extension, $t$ is time and $\lambda_{E}$ is the fluid characteristic relaxation time for the elastocapillary thinning action (Bazilevsky et al. 1990). The Bond number $B o=\rho g D_{m i d}^{2} / \sigma$ may be used to quantify the relative magnitudes of gravitational and surface tension forces, where $\rho$ is the ink density and $g$ is gravitational acceleration; gravitational effects are considered to be unimportant for $B o<O(0.1)$ (McKinley and Tripathi 2000; Clasen 2010). A pynometer was used to obtain fluid density and surface tension was determined dimensionally via the pendant drop method (Bidwell et al. 1964) using images captured with the FastCam high-speed camera and analysed with ImageJ image processing software.

\section{Printing and uniformity analysis}

Flexographic printing of the model inks was carried out on an IGT F1 Printability Tester lab-scale printing press. Solid area printing plates capable of producing full area, featureless coverage (defined in the field as $100 \%$ nominal coverage) at a width of $3 \mathrm{~cm}$ were selected to assist in the analysis of print uniformity. The substrate used was $330 \mu \mathrm{m}$ white polyethylene terephthalate (PET), the anilox volume was $24 \mathrm{~cm}^{3} / \mathrm{m}^{2}$ and the print velocity was $0.2 \mathrm{~m} / \mathrm{s}$. Once printed, samples were dried at room temperature and then digitised with an Epson Perfection V700 scanner at 1200 dots per inch. Quantification of the uniformity of digitised prints was carried out by calculating the total area
Table 2 Important physical properties of the inks: surface tension $(\sigma)$, shear viscosity $(\eta)$, dynamic viscosity $\left(\eta^{\prime}\right)$, elastic modulus $\left(G^{\prime}\right)$ at $1 \mathrm{~Hz}$ and extensional relaxation time $\left(\lambda_{E}\right)$ with respective standard deviations from repeat measurements

\begin{tabular}{llllll}
\hline Fluid & $\sigma(\mathrm{mN} / \mathrm{m})$ & $\eta(\mathrm{mPa} \cdot \mathrm{s})$ & $\eta^{\prime}(\mathrm{mPa} \cdot \mathrm{s})$ & $G^{\prime}\left(\mathrm{Pa} / 10^{-3}\right)$ & $\lambda_{E}(\mathrm{~ms})$ \\
\hline $\mathrm{N}$ & $44.1 \pm 0.5$ & $23.6 \pm 0.2$ & $22.94 \pm 0.01$ & - & - \\
E1 & $44.9 \pm 1.0$ & $23.3 \pm 0.1$ & $22.48 \pm 0.01$ & $1.46 \pm 0.03$ & $68 \pm 2$ \\
E2 & $45.3 \pm 0.9$ & $24.1 \pm 0.5$ & $23.19 \pm 0.02$ & $3.9 \pm 0.1$ & $94 \pm 6$ \\
E3 & $45.2 \pm 0.7$ & $22.6 \pm 0.7$ & $22.48 \pm 0.03$ & $10.2 \pm 0.1$ & $117 \pm 4$ \\
\hline
\end{tabular}


Fig. 2 A print area (left) that has been binarised (centre) and subjected to edge detection routines (right)
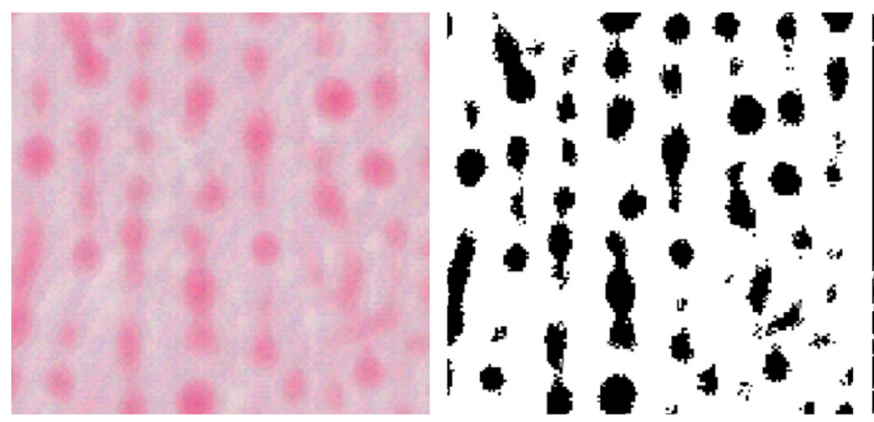

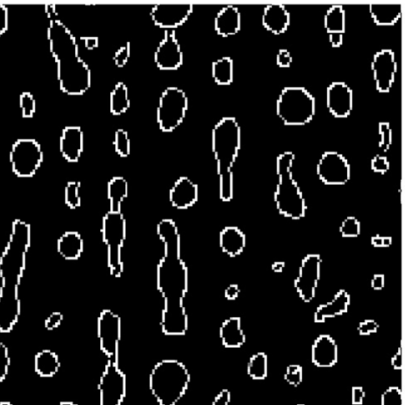

to total circumference ratio of features in a selected area of each print $600 \times 600$ pixels in size. At the edges of the relief printing plate, an air-ink interface exists leading to the formation of ancillary viscous fingers of varying sizes and orientation. Therefore, sample areas were taken sufficiently away from edges to avoid such boundary effects and within the first $10 \mathrm{~cm}$ of the print—during the first inked revolution of the printing plate-for consistent ink transfer. Subsequent image analysis was carried out with Wolfram Mathematica software (Wolfram Research Inc 2015). Total feature area was calculated by binarising the image with Otsu's cluster variance maximisation method (Otsu 1979) while gradient edge detection provided the total feature circumference. Edges in a radius of three pixels were recorded, thus maintaining the detection resolution required for the features of interest while reducing the influence of noise. The ratio of total area to total circumference may therefore serve as a concise quantification of the overall relative size of the print features and thus a measure of its uniformity. The binarisation and edge detection processes are illustrated for a selected sample area in Fig. 2.

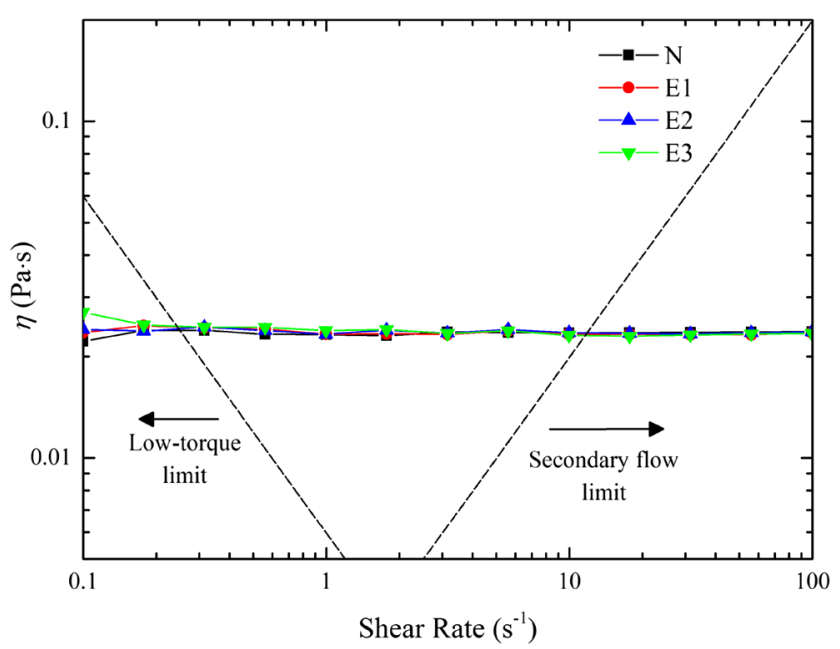

Fig. 3 Shear viscosity of all inks

\section{Results and discussion}

\section{Ink characterisation}

The shear viscosity of each ink is shown in Fig. 3 with low-torque and secondary flow limits marked by dashed lines (Ewoldt et al. 2015), while effects from the latter were not observed. All inks display near constant viscosities with shear rate above the low-torque limit and their mean values, calculated from linear fits of stress and shear rate, are displayed in Table 2. Small-amplitude oscillatory shear measurements taken at a controlled stress of $0.5 \mathrm{~Pa}-$ within the linear viscoelastic range (LVR) of each ink-are displayed in Fig. 4 with the LVR determined by amplitude sweeps at $1 \mathrm{~Hz}$. As the viscosities of the inks are similar, the viscous moduli $G^{\prime \prime}$ are also such, while the elastic moduli $G^{\prime}$ are seen to increase with concentration of PAM. Modulus $G^{\prime}$ of ink N was too low to be accurately measured, as would be expected for a Newtonian sample. This rise in elasticity may be attributed to the increasing presence of the long, flexible polymer chains of the high molecular weight PAM. The low-torque limit of the rheometer was

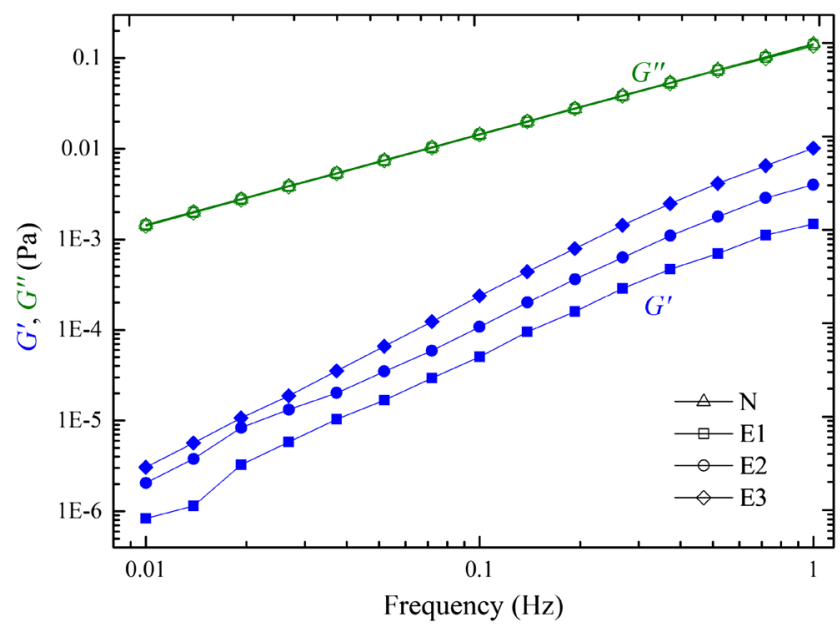

Fig. 4 Elastic modulus $G^{\prime}$ (filled symbols) and viscous modulus $G^{\prime \prime}$ (unfilled symbols) with shear oscillation frequency 
Fig. 5 Sequence of images from CaBER experiments for (a) Newtonian ink $\mathrm{N}$ at 4000 frames/s and (b) the most elastic ink E3, $\lambda_{E}=117$, at 2000 frames/s (a) Ink $\mathrm{N}$
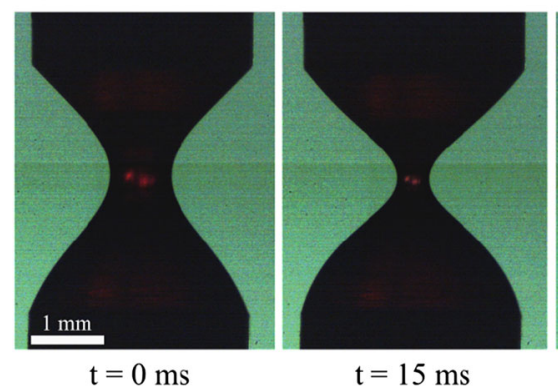

$\mathrm{t}=15 \mathrm{~ms}$

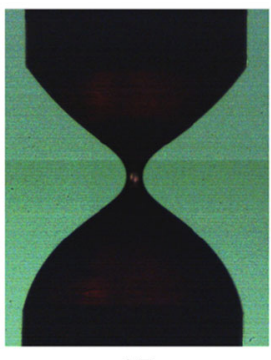

$\mathrm{t}=17 \mathrm{~ms}$

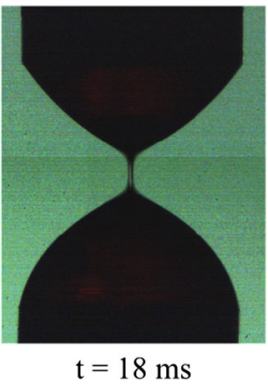

(b) Ink E3

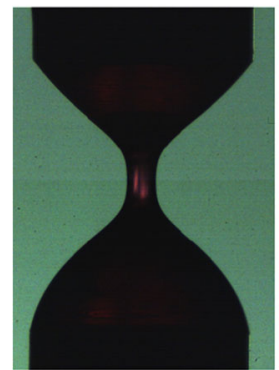

$\mathrm{t}=0 \mathrm{~s}$

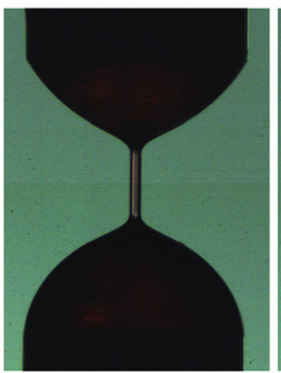

$\mathrm{t}=0.1 \mathrm{~s}$
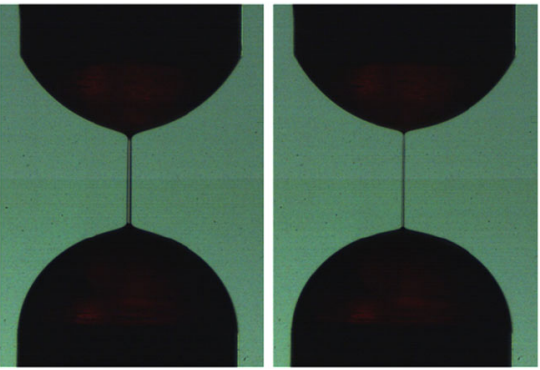

$\mathrm{t}=0.6 \mathrm{~s}$

$\mathrm{t}=0.8 \mathrm{~s}$ surpassed for all measurements, while the raw phase angle reached a maximum of $155^{\circ}$ at the highest frequency and was otherwise below $150^{\circ}$, suggesting reliability regarding inertial affects (Hudson et al. 2017). Such verification was particularly necessary due to the low viscosity of the inks. Furthermore, the dynamic viscosity $\eta^{\prime}=G^{\prime \prime} / \omega$ (Barnes et al. 1989), included in Table 2, shows consistency with $\eta$ and upholds the viscometric similarity of the fluids.

Extensional characterisation using the CaBER technique further demonstrates the trend of increasing elasticity with PAM content. A sequence of images from the filament breakup of ink $\mathrm{N}$ and the most elastic ink E3 are shown in Fig. 5 at selected times with brightness adjusted for visual representation. The typical curvature of the Newtonian filament is evident while the axially symmetric filament formed by ink E3 is characteristic of elastic breakup (Mckinley 2005) and was qualitatively similar for inks E1 and E2. The filament breakup time for each fluid, shown in Fig. 6, increases with PAM content, and the PAMcontaining inks all show exponential decay characteristic of such behaviour before breakup occurs due to the finite extensibility of the polymer chains (Anna and Mckinley 2001). Relaxation times for each fluid, presented in Table 2, were obtained from the fitting of Eq. 2 to the exponential filament decay and therefore elastocapillary regimes of the elastic inks (Sousa et al. 2017) which occurred with Bo $<0.013$, suggesting minimal gravitational effect. The increase of $\lambda_{E}$ with PAM content confirms that its addition greatened ink extensional elasticity. After an initial retardation, likely due to gravitational drainage, ink $\mathrm{N}$ demonstrates a more linear decay where $\mathrm{Bo} \leq$ 0.02 , betraying Newtonian characteristics (McKinley and Tripathi 2000) with an apparent extensional viscosity of $0.11 \pm 0.01 \mathrm{~Pa} \cdot \mathrm{s}$ as described by Eq. 1. This is slightly higher than the Trouton ratio $\eta_{E}=3 \eta$ would predict for Newtonian fluids of such a shear viscosity $\eta$ and may be due to non-negligible inertial and gravitational forces retarding the fluid breakup in light of ink's low viscosity and elasticity (McKinley and Tripathi 2000; Rodd et al. 2005).

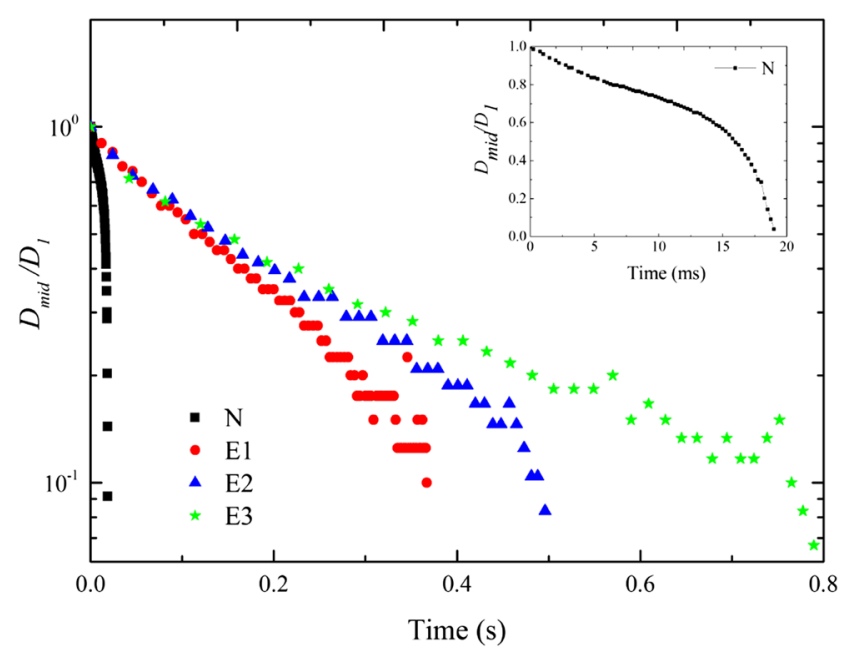

Fig. 6 Typical normalised CaBER filament diameter with time for each ink. Inset: typical Newtonian breakup with a linear normalised diameter scale 
The constant shear viscosity and measurable shear and extensional elasticity of the presently formulated inks E1, E2 and E3 imply they may be considered Boger fluids.

\section{Analysis of printing uniformity}

Non-uniformity consistent with the viscous fingering phenomenon was observed in prints of all inks formulated and representative samples of each print are shown in Fig. 7 with their brightness adjusted to enhance feature visibility. Significant variation can be seen in the nature of the patterns produced from the four inks. Prints from the Newtonian ink exhibit near continuous, periodic striping in the printing direction with a visually counted wavenumber of approximately $2 \mathrm{~mm}^{-1}$. As the ink elasticity is increased, the striping becomes less continuous while the feature orientation remains in the printing direction. Area-circumference feature analysis of $600 \times 600$ pixel areas of the prints conveniently quantifies this uniformity change as the ink elasticity is increased and is plotted with extensional relaxation time $\lambda_{E}$ in Fig. 8 with that of Newtonian ink $\mathrm{N}$ taken to be zero. The area-circumference ratio, fitted with a negative exponential growth, decreases with $\lambda_{E}$, indicative of the apparent reduction in feature size with elasticity. Furthermore, this ratio also decreases with ink elastic modulus $G^{\prime}$, seen inset in Fig. 8 fitted with an exponential decay. Error bars represent the standard deviations of the respective quantities, with those of the calculated ratio reflecting its sensitivity to print variation.

The presence of an elastic component in the ink through the introduction of PAM appears to disrupt the continuous striping during printing causing - at the two lower concentrations-shorter printed features without greatly impacting their width. Such a variation of print uniformity would likely affect the conductivity of a print with a functional ink which may also be directionally dependent, particularly in the case of the striping Newtonian ink. Prints of E3, the ink with the greatest degree of elasticity, however exhibit a somewhat different topology, with significantly narrower features in a more forked

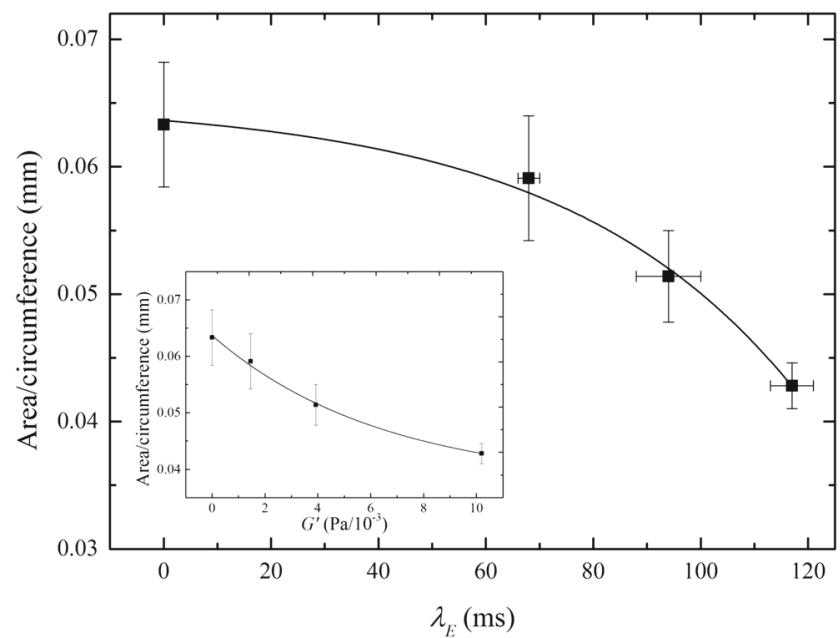

Fig. 8 Total feature area-circumference ratio with extensional relaxation time $\lambda_{E}$ and inset with elastic modulus $G^{\prime}$

configuration that nonetheless continue the trends of decreased area-circumference ratio with elasticity seen in Fig. 8. This convergent forking is present, though to a much lesser extent, in prints of the other inks and also appears to increase with elasticity. The striping disruption may be linked to the elastic influence on filamentation during the separation of the printing plate and the inked substrate, a notion consistent with aforementioned reports regarding roller processes that fluid elasticity leads to smaller filaments (Ercan 2001) and that they are longer and more stable (Fernando and Glass 1988). This is supported by the correlation of extensionally measured elasticity through CaBER, a filament thinning process, and print feature size displayed in Fig. 8 which not only characterises nonuniformity in a roller process but extends it to a real printing scenario on a flexographic press with model inks. Notably, this is the first known reporting of a flexographically printed Boger fluid and given the similarity of the capillary number for the printing of all fluids-between 0.100 and 0.107-the present work highlights the limitations of this parameter for the relation of rheological properties and print

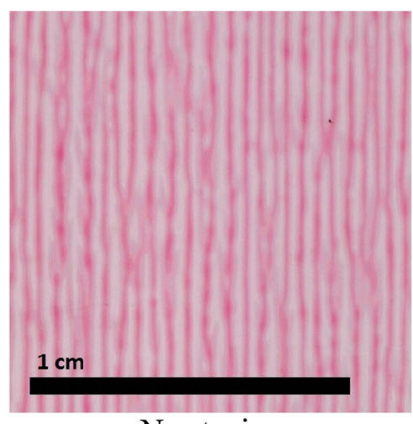

Newtonian

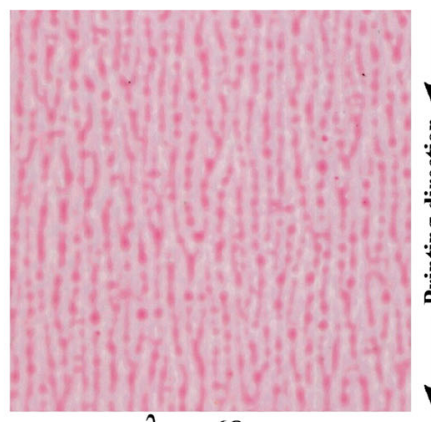

$\lambda_{E}=68 \mathrm{~ms}$
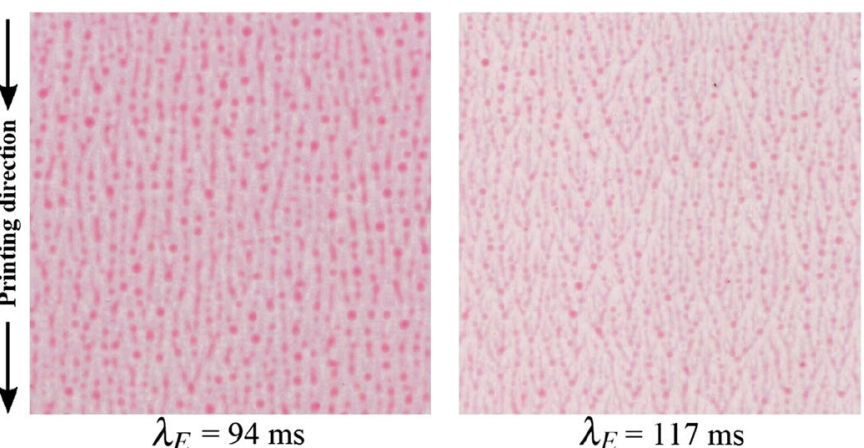

Fig. 7 Sections of print samples for each ink, from left to right: N, E1, E2 and E3 
outcome for elastic inks. Elastocapillary number $E c=$ $\lambda_{E} \sigma / \eta l$, a dimensionless parameter characterising elastic and capillary effects (Mckinley 2005), given here with extensional relaxation time, may therefore be a relevant quantity in this context, where an appropriate length scale $l$ is required. Assuming a $40 \%$ transfer of ink from the anilox to the printing plate (Cherry 2007), a film thickness of $9.6 \mu \mathrm{m}$ would be expected at the nip, implying $E c \sim$ $10^{4}$ for the three elastic inks, increasing with $\lambda_{E}$, and $B o \sim 10^{-5}$ suggesting flow strongly dominated by elastic and surface tension forces. Given the controlled viscosity and surface tension of all four inks, this is consistent with the perception that the print variation observed may be attributed to the effects of ink elasticity on flow in the flexographic process and highlights the possibility of tuning rheological parameters for desired printing patterns.

Further understanding and characterisation of the patterning demonstrated in this work is not only of interest in printed electronics but could also be of use in other applications such as security printing where the random nature of the features may be matched with a digital signature for use on secure documents (Anderson 2008).

\section{Conclusions}

Printable model fluids were formulated and printed via flexography in order to assess the influence of ink elasticity on printing uniformity. Shear and extensional testing confirmed that PVA and PAM solutions were successfully blended to create Boger fluid inks of similar shear viscosity but varying elasticity. Upon printing of all inks, viscous fingering was observed. The presence of ink elasticity disrupted the continuous nature of the striping features formed by the Newtonian ink and resulted in the formation of smaller features on the substrate. This was attributed to the influence of extensional elasticity on the filamentation of the ink during the separation of the printing plate and the inked substrate. The insights presented in this work therefore suggest that control of flexographic ink elasticity in addition to viscosity and surface tension is necessary to ensure optimum layer uniformity, a crucial requisite for electronic device functionality and therefore the emergence of mass-produced printed electronics.

Funding information This research was supported by the EPSRC (UK) grant number EP/M008827/1.

Open Access This article is distributed under the terms of the Creative Commons Attribution 4.0 International License (http:// creativecommons.org/licenses/by/4.0/), which permits unrestricted use, distribution, and reproduction in any medium, provided you give appropriate credit to the original author(s) and the source, provide a link to the Creative Commons license, and indicate if changes were made.

\section{References}

Anderson R (2008) Security engineering: a guide to building dependable distributed systems. Wiley, Hoboken

Anna SL, Mckinley GH (2001) Elasto-capillary thinning and breakup of model elastic liquids. J Rheol 45:115-138

Baker J, Deganello D, Gethin D, Watson T (2014) Flexographic printing of graphene nanoplatelet ink to replace platinum as counter electrode catalyst in flexible dye sensitised solar cell. Mater Res Innov 18(2):86-90

Barnes HA, Hutton JF, Walters K (1989) An introduction to rheology. Elsevier Science Publishers B.V., Amsterdam

Bauman T, Sullivan T, Middleman S (1982) Ribbing instability in coating flows: effect of polymer additives. Chem Eng Commun 14(1-2):35-46

Bazilevsky AV, Entov VM, Rozhkov AN (1990) Liquid filament microrheometer and some of its applications. In: Proceedings of third European rheology conference and golden jubilee meeting of the british society of rheology, pp 41-43

Benson J, Fung CM, Lloyd JS, Deganello D, Smith N, Teng KS (2015) Direct patterning of gold nanoparticles using flexographic printing for biosensing applications. Nanoscale Res Lett 10:127

Bidwell RM, Duran JL, Hubbard GL (1964) Tables for the determination of the surface tensions of liquid metals by the pendant drop method. In: University of California Los Alamos scientific laboratory, pp 1-11

Cherry JA (2007) Ink release characteristics of anilox rolls. PhD thesis, University of Wales Swansea

Clasen C (2010) Capillary breakup extensional rheometry of semidilute polymer solutions. Korea-Australia Rheology Journal 22:331-338

Crooks R, Cooper-White J, Boger DV (2001) The role of dynamic surface tension and elasticity on the dynamics of drop impact. Chem Eng Sci 56(19):5575-5592

Deganello D, Cherry JA, Gethin DT, Claypole TC (2010a) Impact of metered ink volume on reel-to-reel flexographic printed conductive networks for enhanced thin film conductivity. Thin Solid Films 520:2233-2237

Deganello D, Cherry JA, Gethin DT, Claypole TC (2010b) Patterning of micro-scale conductive networks using reel-to-reel flexographic printing. Thin Solid Films 518:6113-6116

Dontula P, Macosko CW, Scriven LE (1998) Model elastic liquids with water-soluble polymers. AIChE J 44:1247-1255

El-din HMN, El-Naggar AWM, Ali FI (2003) Miscibility of poly(vinyl alcohol)/polyacrylamide blends before and after gamma irradiation. Polym Int 52:225-234

Ercan SN (2001) The influence of process parameters on filament size distribution. $\mathrm{PhD}$ thesis, The University of Maine

Ewoldt RH, Johnston MT, Caretta LM (2015) Experimental challenges of shear rheology: how to avoid bad data. Complex Fluids in Biological Systems 7(1):3866-3871

Fernando RH, Glass JE (1988) Dynamic uniaxial extensional viscosity (DUEV) effects in roll application II: polymer blend studies. J. Rheol. 32(2):199-213

Fernando RH, Xing LL, Glass JE (2000) Rheology parameters controlling spray atomization and roll misting behavior of waterborne coatings. Prog Org Coat 40(1-4):35-38

Gaskell PH, Innes GE, Savage MD (1998) An experimental investigation of meniscus roll coating. J Fluid Mech 355:1744

Han SK, Shin DM, Park HY, Junga HW, Hyun JC (2009) Effect of viscoelasticity on dynamics and stability in roll coatings. Eur Phys J Special Topics 166:107-110

Hoath SD, Vadillo DC, Harlen OG, McIlroy C, Morrison NF, Hsiao WK, Tuladhar TR, Jung S, Martin GD, Hutchings IM (2014) 
Inkjet printing of weakly elastic polymer solutions. J NonNewtonian Fluid Mech 205:1-10

Hudson RE, Holder AJ, Hawkins KM, Williams PR, Curtis DJ (2017) An enhanced rheometer inertia correction procedure (ERIC) for the study of gelling systems using combined motor-transducer rheometers. Phys Fluids 29(12):121602

Huzyak P, Koelling K (1997) The penetration of a long bubble through a viscoelastic fluid in a tube. J Non-Newtonian Fluid Mech 71(1-2):73-88

Kipphan H (2001) Handbook of print media: technologies and production methods. Springer, Berlin

Lee TM, Han HS, Kim B, Kwak SW, Noh JH, Kim I (2013) Roll offset printing process based on interface separation for fine and smooth patterning. Thin Solid Films 548:566-571

Lindner A, Bonn D, Poiré EC, Amar MB, Meunier J (2002) Viscous fingering in non-Newtonian fluids. J Fluid Mech 469:237-256

Lindner A, Wagner C (2009) Viscoelastic surface instabilities. C R Phys 245:712-727

Lloyd JS, Fung CM, Deganello D, Wang RJ, Maffeis TGG, Lau SP, Teng KS (2013) Flexographic printing-assisted fabrication of $\mathrm{ZnO}$ nanowire devices. Nanotechnology 24:195602

Malhotra S, Sharma MM (2014) Impact of fluid elasticity on miscible viscous fingering. Chem Eng Sci 117:125-135

Mckinley GH (2005) Visco-elasto-capillary thinning and break-up of complex fluids. Ann Rheol Rev 3:1-49

McKinley GH, Tripathi A (2000) How to extract the Newtonian viscosity from capillary breakup measurements in a filament rheometer. J Rheol 44:653-670

Morrison NF, Harlen OG (2010) Viscoelasticity in inkjet printing. Rheol Acta 49(6):619-632

Otsu N (1979) A threshold selection method from gray-level histograms. IEEE Trans Syst Man Cybern SMC-9(1):62-66

Pangalos G, Dealy JM, Lyne MB (1985) Rheological properties of news inks rheological properties of news inks. J Rheol 29:471-491

Papageorgiou DT (1995) On the breakup of viscous liquid threads. Phys Fluids 7:1529-1544

Rand BP, Genoe J, Heremans P, Poortmans J (2007) Solar cells utilizing small molecular weight organic semiconductors. Prog Photovolt Res Appl 15(2013):659-676

Rodd LE, Scott TP, Cooper-White JJ, McKinley GH (2005) Capillary break-up rheometry of low-viscosity elastic fluids. Appl Rheol $15: 12-27$

Sabadini E, Ungarato RFS, Miranda PB (2014) The elasticity of soap bubbles containing wormlike micelles. Langmuir 30:727-732

Saffman P, Taylor G (1958) The penetration of a fluid into a porous medium or Hele-Shaw cell containing a more viscous liquid. Proc R Soc A 245:312-329
Sauer HM, Bornemann N, Dörsam E (2011) Viscous fingering in functional flexo printing: an inevitable bug? In: Proceedings of the large-area, organic and printed electronics convention (LOPE-C)

Schmidt GC, Bellmann M, Meier B, Hambsch M, Reuter K, Kempa H, Hübler A. C. (2010) Modified mass printing technique for the realization of source/drain electrodes with high resolution. Organic Electronics: physics, materials, applications 11(10):1683-1687

Somalu MR, Yufit V, Brandon NP (2013) The effect of solids loading on the screen-printing and properties of nickel/scandia-stabilizedzirconia anodes for solid oxide fuel cells. Int J Hydrog Energy 38(22):9500-9510

Sousa PC, Vega EJ, Sousa RG, Montanero JM, Alves MA (2017) Measurement of relaxation times in extensional flow of weakly viscoelastic polymer solutions. Rheol Acta 56:11-20

Spaid MA, Homsy GM (1996) Stability of Newtonian and viscoelastic dynamic contact lines. Phys Fluids 8(2):460-478

Sunilkumar K, Rothstein JP (2017) Ink transfer of non-Newtonian fluids from an idealized gravure cell: the effect of shear and extensional deformation. J Non-Newtonian Fluid Mech 243:1626

Tuladhar TR, Mackley MR (2008) Filament stretching rheometry and break-up behaviour of low viscosity polymer solutions and inkjet fluids. J Non-Newtonian Fluid Mech 148:97108

Vlad D, Maher J (2000) Tip-splitting instabilities in the channel Saffman-Taylor flow of constant viscosity elastic fluids. Phys Rev E Stat Phys Plasmas Fluids Relat Interdiscip Topics 61(5B):5439_ 44

Voß C (2002) Analytische modellierung experimentelle untersuchungen und dreidimensionale Gitter-Boltzmann-Simulation der quasistatischen und instabilen Farbspaltung. PhD thesis, Bergische Universität Gesamthochschule Wuppertal

Wang Z, Winslow R, Madan D, Wright PK, Evans JW, Keif M, Rong X (2014) Development of $\mathrm{MnO} 2$ cathode inks for flexographically printed rechargeable zinc-based battery. J Power Sources 268:246-254

Wolfram Research Inc (2015) Mathematica Version 10.1

Yan H, Chen Z, Zheng Y, Newman C, Quinn JR, Dotz F, Kastler M, Facchetti A (2009) A high-mobility electron-transporting polymer for printed transistors. Nature 457:679-687

Yu JH, Fridrikh SV, Rutledge GC (2006) The role of elasticity in the formation of electrospun fibers. Polymer 47:4789-4797

Zevallos GA, Carvalho MS, Pasquali M (2005) Forward roll coating flows of viscoelastic liquids. J Non-Newtonian Fluid Mech 130:96-109 